\title{
Theoretical Overview on (Hidden) Charm in High-Energy Heavy-Ion Collisions
}

\author{
Ralf Rapp $\dagger \S$ and Loic Grandchamp $\ddagger \|$ \\ † NORDITA, Blegdamsvej 17, DK-2100 Copenhagen, Denmark \\ $\ddagger$ Department of Physics, SUNY Stony Brook, NY 11794-3800, U.S.A. and IPN \\ Lyon, 69622 Villeurbanne Cedex, France
}

\begin{abstract}
Recent developments in the theoretical evaluation of charmonium production in ultrarelativistic heavy-ion collisions (URHIC's) are discussed. In particular, the consequences of equilibrium properties of open and hidden charm states - accessible, e.g., in QCD lattice gauge calculations - are assessed. These include abundances as well as formation and dissociation rates of charmonia in both hadronic and quark-gluon matter.
\end{abstract}

\section{Introduction}

Hadrons containing charm quarks play a special role in ultrarelativistic heavy-ion physics $[1,2,3,4,5,6,7,8,9,10,11]$. On the one hand, the charm $(c)$ quark carries a mass, $m_{c} \simeq 1.3 \mathrm{GeV} / \mathrm{c}^{2}$, large enough for its production to be dominated by primordial nucleon-nucleon $(N-N)$ collisions [12]; on the other hand, $m_{c}$ is small enough to allow for significant interactions with surrounding light quarks and gluons, thus providing a rather direct probe of the hot and/or dense matter created in central collisions of heavy nuclei $(A-A)$. This particularly applies for $c-\bar{c}$ bound states (charmonia): based on the production systematics in $p-p$ reactions, where they amount to $\sim 2 \%$ of the total charm $(c \bar{c})$ yield, charmonia have been predicted to be suppressed beyond standard nuclear effects if a deconfined medium is formed in $A$ - $A$ collisions [2]. At the same time, reinteractions of $c$-quarks imply approach towards thermalization, and, in principle, the presence of the backward channel in charmonium dissociation reactions,

$$
X_{1}+J / \psi \rightleftharpoons X_{2}+c+\bar{c}(D+\bar{D}) .
$$

To identify the relevant processes in a heavy-ion reaction, several questions arise, e.g.,

- What are the inelastic charmonium cross sections, and corresponding equilibration times, in the Quark-Gluon Plasma (QGP) and hadronic phase?

- What are the in-medium properties (mass and width) of the charmonium and open charm states in both phases?

- How does the primordially produced number of charmonium states compare to thermal equilibrium abundances?

- What is the significance of the critical temperature $T_{c}$ in the previous items?

$\S$ email: rapp@nordita.dk

|| email: loic@tonic.physics.sunysb.edu 
- Are there large corrections to the expected open charm content of the system, and do $c$-quarks ( $D$-mesons) thermalize?

In this talk we will address (some of) these issues as follows. In Sec. 2 we briefly recall the basic features of open charm production, including new insights from recent RHIC data. In Sec. 3, we first assess in-medium properties of open and hidden charm states from recent lattice QCD results. Second, we elaborate on their possible consequences on the description of charmonium evolution in URIHC's in both QGP and hadronic phases. The main tool will be rate equations with their main ingredients - initial conditions, relaxation times and equilibrium limits - being discussed in some detail. Third, we will comment on statistical hadronization models, in particular how in-medium modifications of charmed hadrons may affect their results. In Sec. 4, we confront various approaches to available data, and in Sec. 5 we conclude.

\section{Open Charm Production}

The production of $c \bar{c}$ pairs in heavy-ion reactions is expected to behave as a hard process, i.e., scale with the number of primordial $N-N$ collisions. While $p$ - $A$ data follow this expectation $[13,14]$, only indirect measurements via semileptonic decays, $c(\bar{c}) \rightarrow l^{+}\left(l^{-}\right) X$ (within charmed hadrons), are available for the $A$ - $A$ case so far.

In $P b-P b$ at SPS energy, $\sqrt{s}=17.3 \mathrm{AGeV}$, NA50 [14] found an enhancement of intermediate-mass ( $I M, M_{\mu \mu}=1.5-2.5 \mathrm{GeV}$ ) dimuon pairs over the expected Drell-Yan and open charm sources, gradually increasing with centrality up to a factor of $\sim 2.5$ in most central events. This excess is well reproduced if an increase in open charm production by a factor of $\sim 3.5$ is postulated. However, underlying mechanisms for such an increase are not easily conceivable. In fact, it has been found $[15,16,17]$ that thermal dimuon radiation from a fireball with reasonable initial temperatures, $T_{i}=200-250 \mathrm{MeV}$, can, maybe more naturally, account for the $I M$ dimuon excess. The experimental resolution of this problem is expected from the NA60 experiment [18].

In $A u$ - $A u$ at RHIC energy, $\sqrt{s}=130,200 \mathrm{AGeV}$, single-electron $\left(e^{ \pm}\right)$transversemomentum $\left(p_{t}\right)$ spectra have been measured at various centralities $[19,20]$. After subtraction of light-hadron decay sources, the remaining spectra can be accounted for by "standard" charm-production extrapolated from $N-N$ event generators. On the one hand, this limits the possibility for an appreciable charm enhancement. On the other hand, it is somewhat surprising that even for central collisions the spectral shape is reasonably well reproduced without any reinteractions, as one would naively expect a softening if charm quarks (partially) thermalize. However, thermalization also implies that $c$-quarks participate in the collective matter expansion. In Ref. [21] it has been shown that the current PHENIX data $[19,20]$ for "charm-like" $e^{ \pm}$are also consistent with the assumption of complete $c$-quark thermalization and collective flow. As discussed below, $c$-quark reinteractions have important consequences for charmonium production; the obvious observable to disentangle the two extremes is the $c$-quark elliptic flow [21].

\section{Charmonium in QCD Matter}

\subsection{Lattice QCD Results}

Lattice QCD calculations for the finite- $T$ free energy of a heavy-quark pair [22],

$$
F_{Q \bar{Q}}(r, T)=V_{Q \bar{Q}}(r, T)-T S_{Q \bar{Q}}(r, T)
$$



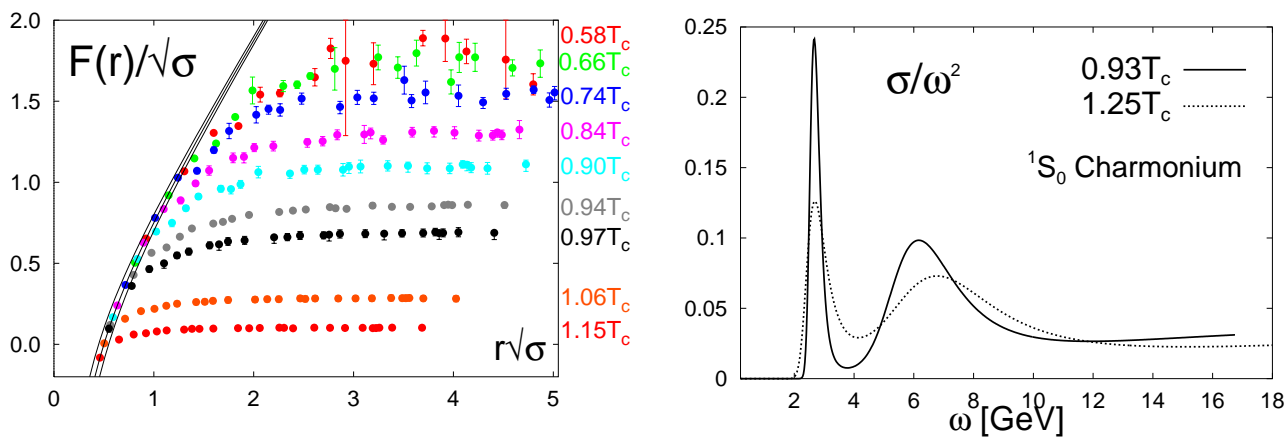

Figure 1. Left panel: free energy of a heavy-quark pair from unquenched $\left(N_{f}=2+1\right)$ lattice calculations [22] as a function of $Q-\bar{Q}$ separation $r$ for various temperatures $T$. Right panel: quenched lattice results [24] for the $\eta_{c}$ spectral function above and below $T_{c}$.

including dynamical fermions, are displayed in the left panel of Fig. 1. The decrease of the asymptotic plateau value, $F_{Q \bar{Q}}(T, r \rightarrow \infty)$, can be attributed to an in-medium reduction of the open charm threshold in the heavy-quark potential, $V_{Q \bar{Q}}$, and has been used to infer "dissociation" temperatures $T_{d i s s}<T_{c}$ for $\psi^{\prime}$ and $\chi$ states, as well as $T_{\text {diss }} \simeq 1.1 T_{c}$ for the $J / \psi$ [23]. It should be emphasized that the temperature dependence of the plateau is remarkably continuous, even across $T_{c}$; this has interesting implications which we will return to below. If bound states move above the threshold for spontaneous decay, it does, however, not necessarily mean that they disappear from the spectrum, but they can rather survive as resonances $\uparrow$. This is indeed found in two recent (quenched) lattice calculations [24, 25], where reconstructed $\eta_{c}$ and $J / \psi$ spectral functions exhibit well-defined states up to $T \simeq 1.5 T_{c}$, see right panel of Fig. 1. The masses appear to be essentially $T$-independent (quite contrary to the open charm states), whereas the widths, $\Gamma_{\eta_{c}, \psi}$, increase significantly, to about $0.2-0.5 \mathrm{GeV}$ slightly above $T_{c}[24,25]$. This is not much different from lifetimes obtained using gluon-induced $J / \psi$ dissociation cross sections [26] at comparable temperatures.

\subsection{Rate Equations in the Quark-Gluon Plasma}

Let us now turn to the description of the time evolution of $J / \psi$ abundances relevant for heavy-ion reactions, first focusing on the QGP phase. A corresponding rate equation for the number of $J / \psi$ mesons in a system (fireball) of volume $V_{F B}$, $N_{\psi}(\tau)=n_{\psi}(T) V_{F B}(\tau)\left(n_{\psi}(T): J / \psi\right.$ density $)$, is of the form

$$
\frac{d}{d \tau} N_{\psi}=-N_{\psi} L(\tau)+G(\tau)=-\frac{1}{\tau_{\psi}}\left[N_{\psi}(\tau)-N_{\psi}^{e q}(\tau)\right],
$$

which applies as long as a well-defined $J / \psi$ state exists, i.e., $\tau_{\psi}^{-1}(T)=\Gamma_{\psi}(T) \ll m_{\psi}$. The second equality in Eq. (3) follows under the simplifying assumption that the surrounding partons (including the charm quarks) are in thermal equilibrium. The

I note that, in a heat bath, the distinction between bound state and resonance becomes immaterial; all information (mass and width) is encoded in the spectral function. 

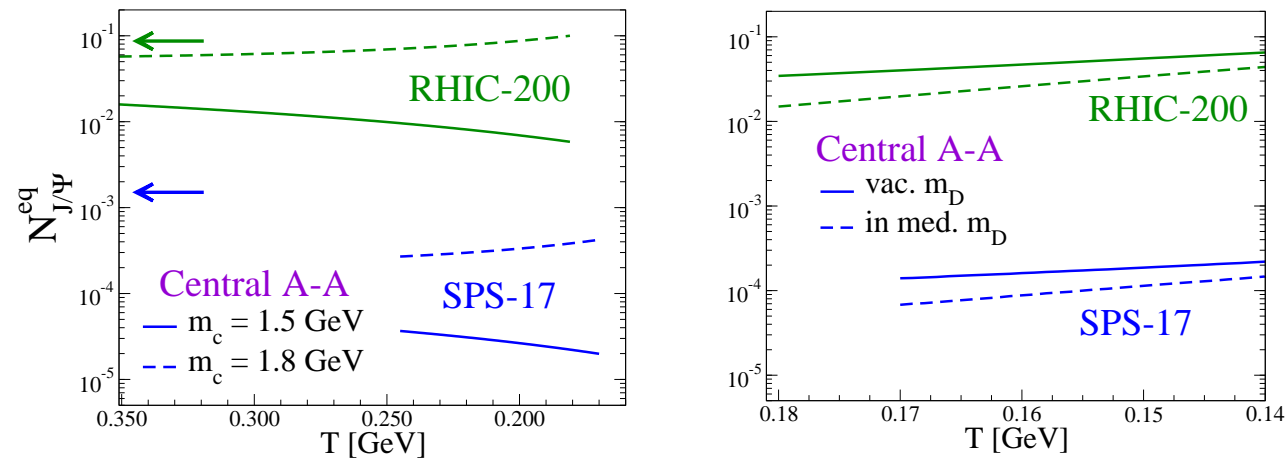

Figure 2. Left panel: Equilibrium $J / \psi$ abundances (assuming $m_{\psi}(T) \equiv m_{\psi}^{v a c}$ ) in an isotropic, adiabatically expanding Quark-Gluon Plasma at fixed (anti-) charmquark number, $N_{c \bar{c}}$, resembling conditions at RHIC and SPS for two values of the charm-quark mass. The arrows mark the numbers, $N_{\psi}^{\text {prim }}$, of $J / \psi$ 's produced in primordial (hard) $N-N$ collisions (as extrapolated from elementary $p-p$ reactions). Right panel: $J / \psi$ equilibrium numbers in the hadronic phase (under equivalent conditions as in the left panel) using free and in-medium charmed hadron masses.

charm-quark and $J / \psi$ equilibrium densities are then given by

$$
\begin{aligned}
& n_{c}\left(T, \gamma_{c}\right)=n_{\bar{c}}\left(T, \gamma_{c}\right)=6 \gamma_{c} \int \frac{d^{3} q}{(2 \pi)^{3}} f^{c}\left(m_{c} ; T\right) \\
& n_{\psi}^{e q}\left(T, \gamma_{c}\right)=3 \gamma_{c}^{2} \int \frac{d^{3} q}{(2 \pi)^{3}} f^{\psi}\left(m_{\psi} ; T\right) .
\end{aligned}
$$

Here we have already incorporated the possibility of chemical off-equilibrium by introducing the charm-quark fugacity $\gamma_{c}$. In practice, the latter will be matched to the total number, $N_{c \bar{c}}$, of $c \bar{c}$ pairs produced in hard (primordial) $N-N$ collisions [27],

$$
N_{c \bar{c}}=\frac{1}{2} N_{o p} \frac{I_{1}\left(N_{o p}\right)}{I_{0}\left(N_{o p}\right)}+V_{F B}(T) \gamma_{c}(T)^{2} \sum_{X=\eta_{c}, \psi, \ldots} n_{X}(T)
$$

with $N_{o p}=2 V_{F B}(T) \gamma_{c}(T) n_{c}(T)$. Fig. 2 (left panel) shows equilibrium $J / \psi$ numbers in the QGP under conditions expected for central $\mathrm{Pb}-\mathrm{Pb} / \mathrm{Au}-\mathrm{Au}$ collisions at SPS/RHIC. One observes a large sensitivity to the (in-medium) charm-quark mass, with larger values for $m_{c}$ requiring a larger fugacity $\gamma_{c}$ (at fixed $N_{c \bar{c}}$ ), and thus entailing a larger equilibrium abundance of charmonium states.

Besides the equilibrium $J / \psi$ number the other essential quantity figuring into Eq. (3) is the (chemical) relaxation time $\tau_{\psi}$ (related to processes given in Eq. (1)). In a perturbative framework, it is determined in terms of parton-induced $J / \psi$ breakup (inelastic) cross sections, $\sigma_{\psi}^{\text {inel }}$, via convolution over thermal parton distributions $f^{i}$,

$$
\tau_{\psi}^{-1}=\int \frac{d^{3} k}{(2 \pi)^{3}} \sum_{i=q, \bar{q}, g} f^{i}(k ; T) \sigma_{i \psi}^{i n e l} .
$$

The use of the standard gluodissociation cross section [1,28], depicted in the upper left panel of Fig. 3, leads to relaxation times $\tau_{\psi} \geq 10 \mathrm{fm} / \mathrm{c}$ for temperatures $T \leq 200 \mathrm{MeV}$, steeply falling to below $1 \mathrm{fm} / \mathrm{c}$ at $T \geq 300 \mathrm{MeV}$. If, however, according to the lattice data in Fig. 1, the threshold energy of the outgoing open charm states is substantially 

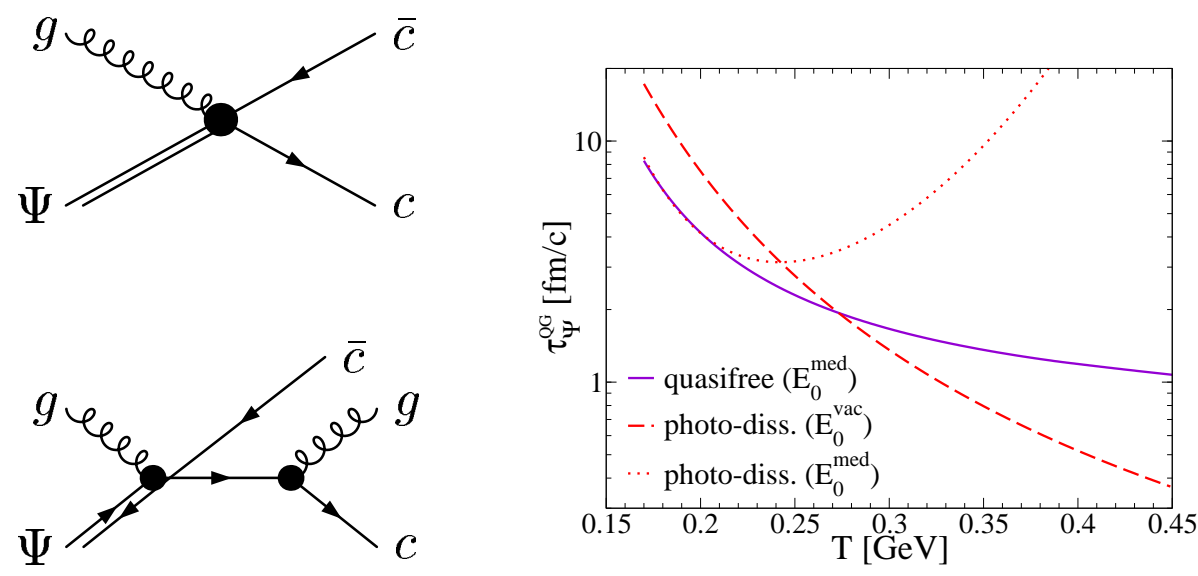

Figure 3. Left panel: parton-induced $J / \psi$ dissociation processes (upper panel: the equivalent of photodissociation induced by gluons; lower panel: quasifree destruction); Right panel: Corresponding $J / \psi$ relaxation times.

reduced and approaches the $J / \psi$ mass, this process becomes kinematically unfavorable, see dotted line in right panel of Fig. 3. Under these circumstances, "quasifree" dissociation [26], which is characterized by an extra outgoing parton (lower left panel in Fig. 3), is much more efficient, cf. solid line in the right panel of Fig. 3.

Nonperturbative approaches to the dissociation of $J / \psi$ 's in a QGP often involve the notion that also " $D$ "-meson states survive the phase transition $[8,29]$; e.g., in constituent-quark based pictures [29], D-mesons exhibit the "Mott" effect, i.e., become broad resonances in the $c-\bar{q}$ continuum above $T_{c}$, thus opening phase space for $J / \psi \rightarrow " D "+" \bar{D} "$ decays.

The final ingredient needed for an actual solution of the rate equation is the initial condition, $N_{\psi}^{0}$, usually taken from hard production systematics (modified by initial-state nuclear absorption to account for "pre-equilibrium" dynamics). One can then specify under which conditions the gain term in Eq. (3) can be neglected in applications to heavy-ion reactions, namely: either (i) $\tau_{\psi} \gg \tau_{Q G P}\left(\tau_{Q G P}\right.$ : lifetime of the QGP phase) and $N_{\psi}^{0} \gg N_{\psi}^{e q}$ or (ii) no $J / \psi$ state is supported in the QGP. The arrow markers in the left panel of Fig. 2 indicate how the expected primordial production numbers of $J / \psi$ 's compare to the thermal equilibrium levels. Whereas at SPS energies $N_{\psi}^{e q} \ll N_{\psi}^{\text {prim }}$, this no longer holds for RHIC energies, i.e., a neglection of the gain term appears to be justified at SPS, but this is not obvious at RHIC.

\subsection{Hadronic Matter: Statistical Production and in-Medium Effects}

Based on the success of thermal models to describe the production of hadrons containing $u, d$ and $s$ quarks [30], it has been suggested [27] that also charmed hadrons form by statistical hadronization (of pre-existing $c, \bar{c}$ quarks) according to thermal weights at $T_{c}$. This, in particular, has been applied to charmonium states [27, 31, 32] by employing Eqs. (5) and (6) but with the fugacity $\gamma_{c}$ determined by the hadronic open charm states, $N_{o p}=V_{F B}\left(T_{c}\right) \gamma_{c} \sum_{\alpha} n_{\alpha}\left(T, \mu_{B}\right)$, where $\alpha=D, \bar{D}, D^{*}, \bar{D}^{*}, \Lambda_{c}$, 


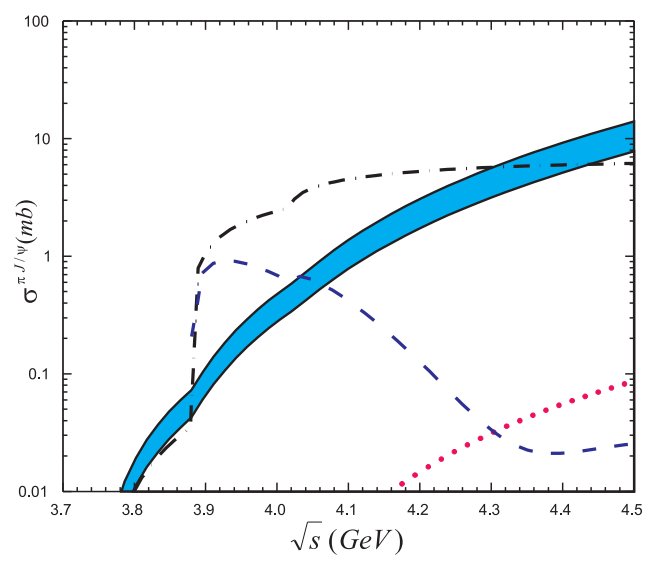

Figure 4. $\pi J / \psi \rightarrow D \bar{D}, D^{*} \bar{D}, D \bar{D}^{*}$ dissociation cross sections in various approaches; band: $\mathrm{QCD}$ sum rules [34], dotted line: short-distance QCD [42], dashed-dotted line: meson-exchange [40], dashed line: nonrelativistic constituent quark model [36].

$\ldots$, runs over all known open charm hadrons, and $V_{F B}\left(T_{c}\right)$ is the hadronic fireball volume after completion of the phase transition (see also Ref. [33]).

In the language of the rate Eq. (3), the statistical hadronization approach corresponds to having reached complete thermal and (relative) chemical equilibrium at $T_{c}$. Interactions in the subsequent hadronic phase may still alter the charmonium abundances, as determined by inelastic hadronic reactions of type $\pi J / \psi \leftrightarrow D \bar{D}^{*}+$ c.c., $\rho J / \psi \leftrightarrow D \bar{D}$, etc., which have been intensely investigated, mostly with focus on the dissociation direction. An example of the current status is shown in Fig. 4, taken from Ref. [34]: the band, which is the main result of that work, represents the pion-induced dissociation cross-section as assessed within a QCD sum rule analysis. It is in reasonable agreement with evaluations from both constituentquark [35, 36] and meson-exchange models $[37,38,39,40,41]$ (once soft form factors with cutoffs $\Lambda \leq 1 \mathrm{GeV}$ are included), but significantly above earlier short-distance QCD calculations [42] based on gluodissociation [28] folded with the (very soft) gluon distribution functions within light hadrons. $\rho$ - [36, 38] and nucleon-induced [43] break-up cross sections tend to be somewhat larger. But even under rather extreme assumptions, e.g., an average cross section of $1 \mathrm{mb}$ and a total hadron density $n_{\text {had }}=3 n_{0} \simeq 0.5 \mathrm{fm}^{-3}$, the chemical relaxation time, $\tau_{\psi}=\left[\langle\sigma v\rangle n_{h a d}\right]^{-1} \simeq 30-40 \mathrm{fm} / \mathrm{c}$, is well below typical hadronic fireball lifetimes.

The situation may change if additional medium effects are present, most notably reduced $D$-meson masses, as indicated by the lattice results in the left panel of Fig. 1, QCD sum rule estimates [44] or model calculations [45]. In a constituent quark picture, such modifications are attributed to the light quarks losing their mass, thus directly reflecting on (partial) chiral symmetry restoration. From a practical point of view, the lowering of the $D \bar{D}$ threshold has several consequences, e.g.: (i) excited charmonia may move above threshold (well) below $T_{c}$ [23], (ii) increased phase space accelerates the inelastic reactions (although wave function nodes in excited charmonia can lead to strong deviations from naive extrapolations [46]) (iii) the equilibrium abundance of charmonia in hadronic matter decreases (at fixed $N_{c \bar{c}}$ ). The latter effect is illustrated 
in the right panel of Fig. 2, and is completely analogous to varying $m_{c}$ in the QGP, see discussion after Eq. (6). Even a moderate reduction of the $D$-meson masses by $80 \mathrm{MeV}$ at $T_{c}=170 \mathrm{MeV}$ lowers the $J / \psi$ equilibrium level by a factor of 2 . The other remarkable feature of the hadronic equilibrium $J / \psi$ numbers is that they increase with decreasing temperature - again a consequence of the increasing $\gamma_{c}$ at fixed $N_{c \bar{c}}$ together with $M_{\psi}<2 m_{D}{ }^{+}$. For heavy-ion reactions this implies that, should the $J / \psi$ number reach equilibrium at or around $T_{c}$, subsequent hadronic reactions would favor additional formation over dissociation (this could be even more relevant for excited charmonium states which only exist in hadronic matter). Such a behavior has indeed been observed in kinetic [47] and transport [48, 49] model calculations.

\subsection{Continuity?}

Before we turn to quantitative model comparisons to heavy-ion data, let us reiterate ramifications of the lattice QCD results [22] for the heavy-quark free energy (left panel of Fig. 1) on the $J / \psi$ evolution within the rate-equation framework. As mentioned above, the asymptotic plateau, $F_{\bar{c}}(T, r \rightarrow \infty)$, suggests a gradual decrease of the open charm threshold with increasing temperature [23], which is surprisingly continuous even when passing through the phase transition. At the same time, the $J / \psi$ mass is essentially constant. The latter implies that, in thermal and chemical equilibrium $\left(\gamma_{c}=1\right)$, the $J / \psi$ density, Eq. (5), only depends on temperature and thus carries no notion of phase transition dynamics. Of course, in a finite system, the latent heat released in going from quark-gluon to hadronic degrees of freedom would imply the total $J / \psi$ number to increase according to the volume expansion at $T_{c}$. However, this is not the situation envisaged in a heavy-ion reaction, where, in addition, one expects the total charm-quark number, $N_{c \bar{c}}$, to be approximately constant, encoded in the chemical off-equilibrium parameter $\gamma_{c} \neq 1$. Hence, through Eqs. (5) and (6), $J / \psi$ abundances are coupled to $N_{c \bar{c}}$ and spectral distributions of open-charm states (figuring into the densities), parametrically as $N_{\psi} \propto n_{\psi} /\left(V_{F B} n_{o p}^{2}\right)$ with $n_{o p}=2 n_{c}\left(m_{c}^{*}, T\right)$ or $\sum_{\alpha=D, D^{*}, \ldots} n_{\alpha}\left(m_{\alpha}^{*}, T\right)$, corresponding to QGP or hadron gas (HG), respectively. It furthermore seems conceivable that the open-charm spectrum - not only the lowest level - changes rather continuously through $T_{c}$, and therefore $n_{o p}^{Q G P} \simeq n_{o p}^{H G}$ (e.g., with in-medium charm-quark masses $m_{c}^{*}>m_{c}=1.15-1.35 \mathrm{GeV} / \mathrm{c}^{2} \mathrm{due}$ to heavy-quark thermal correlation energies in the QGP). In this case, the equilibrium charmonium numbers decrease during the phase transition according to the volume increase at $T_{c}$, quite contrary to the chemical-equilibrium scenario $\left(\gamma_{c} \equiv 1\right)$.

\section{Model Approaches at SPS and RHIC}

At SPS energies, the interpretation of the NA50 data $[50,51]$ is still under debate. On the one hand, "comover" models [52] with a constant dissociation cross section of $\sigma_{c o}^{\text {inel }} \simeq 1 \mathrm{mb}$ are able to explain the observed suppression. One should note, however, that the underlying comover densities reached in the early stages of a collision albeit not necessarily thermal - correspond to energy densities well above critical ones obtained from lattice QCD. On the other hand, the recent idea [27, 31] of statistical coalescence of $c$ and $\bar{c}$ quarks at $T_{c}$ has stimulated further activity. One of its strongholds is the $\psi^{\prime} / \psi$ ratio [27], which experimentally [53] tends to saturate at the thermal value in sufficiently central $A$ - $A$ collisions. To describe the $J / \psi$ data by

+ In the academic limit $T \rightarrow 0$, all $c \bar{c}$ pairs would be in the lowest possible state, i.e., $\eta_{c}$ mesons! 

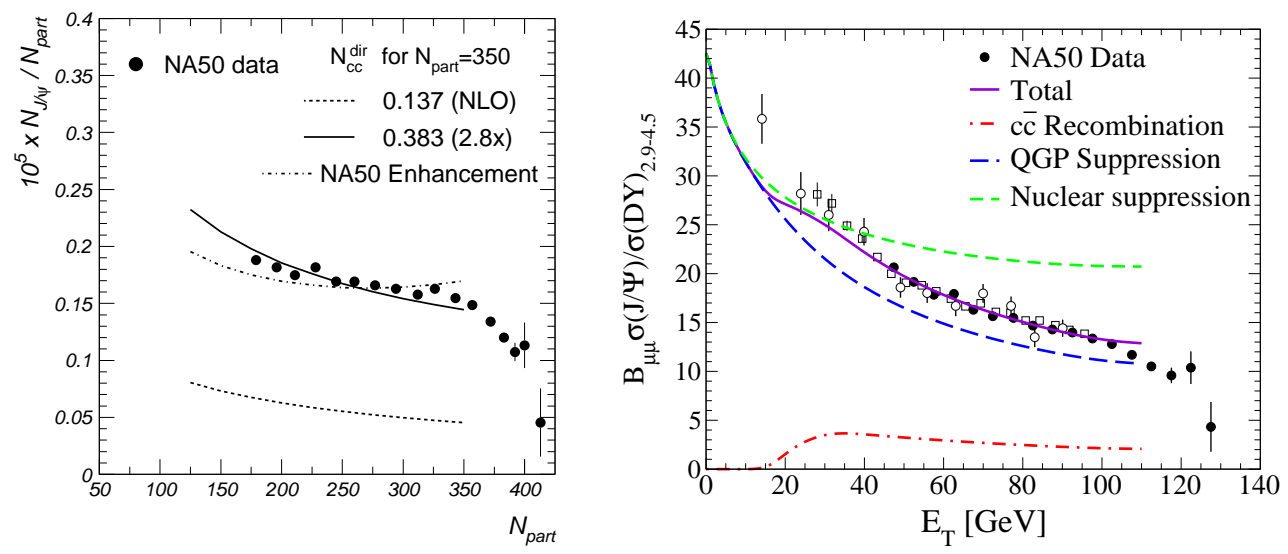

Figure 5. Recent model approaches to NA50 $\mathrm{J} / \psi$ data [50] from $P b-P b$ collisions at SPS $\left(\sqrt{s_{N N}}=17.3 \mathrm{GeV}\right)$. Left panel: statistical coalescence [32]; right panel: two-component model [26].

statistical production alone (assuming all primordial $J / \psi$ 's to be suppressed or not to form), requires an open charm enhancement by a factor of $\sim 3$ (in line with the excess inferred from $I M$ dimuon spectra, cf. the discussion in Sec. 2), see left panel of Fig. 5. In Ref. [26] a two-component approach, combining suppression mechanisms (nuclear, QGP and hadronic) with statistical production at $T_{c}$, has been suggested which allows to incorporate peripheral $A-A$ and $p$ - $A$ collisions and refrains from introducing an anomalous charm enhancement. In this case, QGP suppression is identified as the key feature at SPS, see right panel of Fig. 5.

Let us finally confront predictions for RHIC energies with (preliminary) data from PHENIX [54]. The left panel of Fig. 6 shows the statistical model yields [27, 32], illustrating inherent uncertainties due to the thus far not accurately known opencharm cross section. In the right panel of Fig. 6, the results of the two-component model $[26,55]$ indicate that for (semi-) central collisions at RHIC the thermal production at $T_{c}$ indeed dominates over the primordial contribution, as the latter is subject to strong QGP suppression [11]. This has interesting consequences for the excitation function from SPS to RHIC energies [26]. The upper solid line in the right panel of Fig. 6 is obtained from calculations [56] solving the full rate Eq. (3), using the gluodissociation cross section with a vacuum $J / \psi$ mass and width. Although $\Gamma_{\psi}$ is probably increased, the idea of a $J / \psi$ state in the QGP is supported by lattice QCD, recall the right panel of Fig. 1. The $J / \psi$ formation in this calculation (which does not invoke a hadronic phase) indeed occurs preferentially in the early QGP stages which accounts for most of the difference to the 2-component model (where, in turn, $J / \psi$ formation in the QGP has been neglected). Predictions in line with the current data were also made within a multiphase transport model in Ref. [48], see also Ref. [49] for a recent analysis. In both these calculations, secondary $J / \psi$ formation, i.e., the backward reaction in Eq. (1), again plays a decisive role. In Ref. [57] it has been argued, that a reduced nuclear absorption could be consistent with the data without invoking any regeneration processes. 

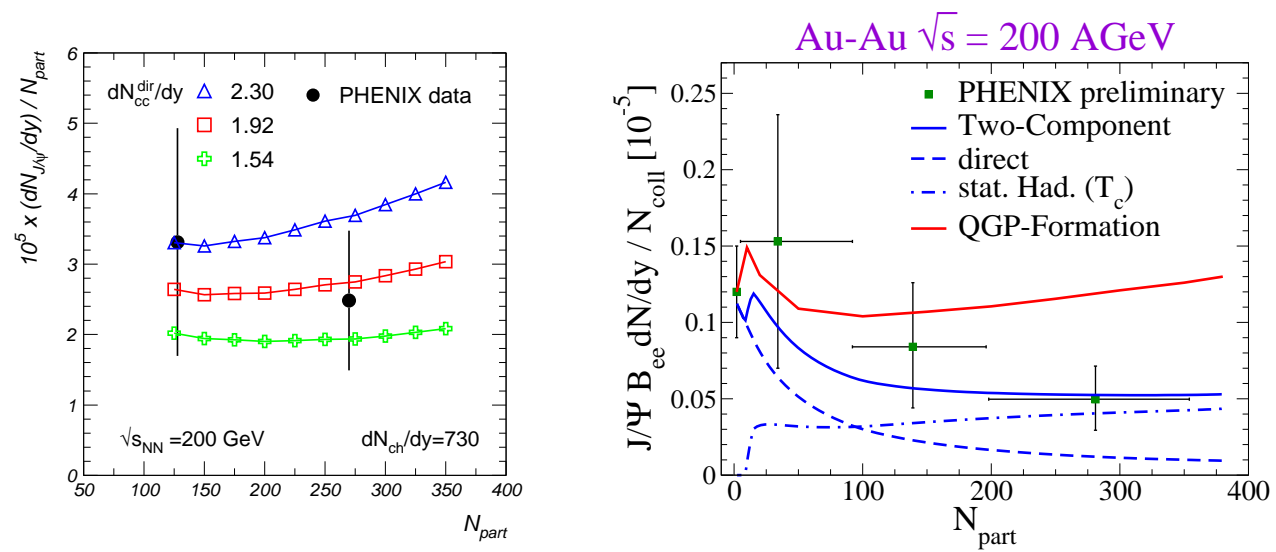

Figure 6. Model predictions for $J / \psi$ production at RHIC $\left(\sqrt{s_{N N}}=200 \mathrm{GeV}\right)$ compared to preliminary PHENIX data [54]. Right panel: statistical hadronization [32]; left panel: dynamical approaches (lower solid line: two-component model [26], upper solid line: yields from solving rate equations in a QGP [56]).

\section{Conclusion}

About 20 years after the first ideas on utilizing charmonium states as a probe of hot and dense QCD matter in heavy-ion collisions, important insights continue to emerge. In addition to experimental data, lattice QCD calculations are now providing valuable information for theoretical model approaches, most notably the survival of (some) charmonia as resonance states in the QGP as encoded in pertinent spectral functions, and the smooth behavior of open charm thresholds through both hadronic and early QGP phases, across $T_{c}$. We attempted to infer consequences thereof in the heavy-ion environment, e.g., the necessity to include charmonium formation reactions, the behavior of $J / \psi$ equilibrium levels across the hadronization transition and its interplay with in-medium effects on open charm states. The current high-energy frontier at RHIC (and future LHC) implies, for the first time, multiple production of $c \bar{c}$ pairs, as well as more favorable conditions for their reinteractions. If charm quarks indeed thermalize in the course of an $A$ - $A$ collision, the equilibrium level of charmonium states being comparable to primordial production, necessarily entails significant regeneration. Whether such a regime has already been reached with RHIC energies cannot be decided from the present $J / \psi$ and single- $e^{ \pm}$data. Future measurements in the upcoming RHIC runs (including open charm observables and possibly excited charmonia), in connection with further theoretical developments, will hopefully hold (some) answers.

\section{Acknowledgement}

One of us (RR) thanks the organizers of the conference for the invitation to this very informative and pleasant meeting. We are grateful to D. Blaschke, C.M. Ko and E.V. Shuryak for stimulating discussions. This work was supported in part by the U.S. DOE under contract no. DE-FG02-88ER40388. 
Theoretical Overview on (Hidden) Charm in High-Energy Heavy-Ion Collisions

\section{References}

[1] Shuryak E V 1978 Sov. J. Nucl. Phys. 28408

[2] Matsui T and Satz H 1986 Phys. Lett. B 178416

[3] Karsch F and Petronzio R 1987 Phys. Lett. B 193105

[4] Blaizot J-P and Ollitrault J-Y 1987 Phys. Lett. B 199499

[5] Svetitsky B 1988 Phys. Rev. D 372484

[6] Röpke G, Blaschke D and Schulz H 1988 Phys. Lett. B 202479

[7] Gavin S, Gyulassy G and Jackson A 1988 Phys. Lett. B 207257

[8] Hansson T H, Lee S H and Zahed I 1988 Phys. Rev. D 372672

[9] Kharzeev D 1998 Nucl. Phys. A 638279

[10] Gerschel C and Hüfner J 1999 Ann. Rev. Nucl. Part. Sci 49255

[11] Vogt R 1999 Phys. Rep. 310197

[12] Lévai P, Müller B and Wang X N 1995 Phys. Rev. C 513326

[13] E789 Collaboration (Leitch M J et al) 1994 Phys. Rev. Lett. 722542

[14] NA38 and NA50 Collaborations (Abreu M C et al) 2000 Eur. J. Phys. C 14443

[15] Rapp R and Shuryak E V 2000 Phys. Lett. B 47313

[16] Gallmeister K, Kämpfer B and Pavlenko O P 2000 Phys. Lett. B 47320

[17] Kvasnikova I, Gale C and Srivastava D K 2002 Phys. Rev. C 65064903

[18] NA60 Collaboration (Oppedisano C et al) 2003 these proceedings

[19] PHENIX Collaboration (Adcox K et al ) 2002 Phys. Rev. Lett. 88192303

[20] PHENIX Collaboration (Averbeck R et al) 2003 Nucl. Phys. A $\mathbf{7 1 5} 695 \mathrm{c}$

[21] Batsouli S, Kelly S, Gyulassy M and Nagle J L 2003 Phys. Lett. B 55726

[22] Karsch F, Laermann E and Peikert A 2001 Nucl. Phys. B 605579

[23] Digal S, Petreczky P and Satz H 2001 Phys. Lett. B 51457

[24] Datta S, Karsch F, Petreczky P and Wetzorke I 2002 LANL arXiv hep-lat/0208012

[25] Umeda T, Nomura K and Matsufuru H 2002 LANL arXiv hep-lat/0211003

[26] Grandchamp L and Rapp R 2001 Phys. Lett. B 523 60; 2002 Nucl. Phys. A 709415

[27] Braun-Munzinger P and Stachel J 2000 Phys. Lett. B 490 196; 2001 Nucl. Phys. A 690 119c

[28] Bhanot G and Peskin M E 1979 Nucl. Phys. B 156391

[29] Blaschke D, Burau G, Barnes T, Kalinovsky Yu and Swanson E S 2002 arXiv hep-ph/0210265

[30] Braun-Munzinger P, Redlich K and Stachel J 2003 arXiv nucl-th/0304013

[31] Gorenstein M I, Kostyuk A P, Stöcker H and Greiner W 2001 Phys. Lett. B 509277

[32] Andronic A, Braun-Munzinger P, Redlich K and Stachel J 2003 arXiv nucl-th/0303036

[33] Gaździcki M and Gorenstein M I 1999 Phys. Rev. Lett. 834009

[34] Durães F O, Kim H, Lee S H, Navarra F S and Nielsen M 2002 arXiv nucl-th/0211092

[35] Martins K, Blaschke D and Quack E 1995 Phys. Rev. C 512723

[36] Wong C-Y, Swanson E S and Barnes T 2001 Phys. Rev. C 65014903

[37] Matinyan S G and Müller B 1998 Phys. Rev. C 582994

[38] Haglin K and Gale C 2001 Phys. Rev. C 63065201

[39] Lin Z W and Ko C M 2000 Phys. Rev. C 62034903

[40] Oh Y, Song T and Lee S H 2001 Phys. Rev. C 63034901

[41] Navarra F S, Nielsen M and Robilotta M R 2001 Phys. Rev. C 64021901

[42] Kharzeev D and Satz H 1994 Phys. Lett. B 334155

[43] Liu W, Ko C M and Lin Z W 2001 Phys. Rev. C 65015203

[44] Hayashigaki A 2000 Phys. Lett. B 48796

[45] Tsushima K, Lu D H, Thomas A W, Saito K and Landau R H 1999 Phys. Rev. C 592824

[46] Friman B, Lee S H and Song T 2002 Phys. Lett. B 548153

[47] Braun-Munzinger P and Redlich K 2000 Eur. J. Phys. C 16519

[48] Zhang B, Ko C M, Li B-A, Lin Z-W and Pal S 2002 Phys. Rev. C 65054909

[49] Bratkovskaya E L, Cassing W and Stöcker H 2003 arXiv nucl-th/0301083 (Phys. Rev. C in print)

[50] NA50 Collaboration (Abreu M C et al ) 2000 Phys. Lett. B 477 28; 1999 Phys. Lett. B 450456

[51] NA50 Collaboration (Ramello L et al) 2003 Nucl. Phys. A 715243

[52] Capella A, Ferreiro E G and Kaidalov A B 2000 Phys. Rev. Lett. 852080

[53] NA50 Collaboration (Ramello L et al ) 1998 Nucl. Phys. A 638261

[54] PHENIX Collaboration (Frawley A D et al) 2003 Nucl. Phys. A 715687

[55] Grandchamp L and Rapp R 2003 Nucl. Phys. A 715545

[56] Thews R L, Schroedter M and Rafelski J 2001 Phys. Rev. C 63 054905; J. Phys. G 27715

[57] Capella A and Sousa D 2003 arXiv nucl-th/0303055 